\title{
The influence of wall roughness on bubble drag reduction in Taylor-Couette turbulence
}

\author{
Ruben A. Verschoof ${ }^{1}$, Dennis Bakhuis ${ }^{1}$, Pim A. Bullee ${ }^{1}$, \\ Sander G. Huisman ${ }^{1}$, Chao Sun ${ }^{2,1, \dagger}$ and Detlef Lohse ${ }^{1,3, \dagger}$ \\ ${ }^{1}$ Physics of Fluids, Max Planck Institute for Complex Fluid Dynamics, MESA+ Institute and \\ J. M. Burgers Center for Fluid Dynamics, University of Twente, PO Box 217, \\ 7500 AE Enschede, The Netherlands \\ ${ }^{2}$ Center for Combustion Energy, Key Laboratory for Thermal Science and Power Engineering of \\ Ministry of Education, Department of Energy and Power Engineering, Tsinghua University, \\ 100084 Beijing, China \\ ${ }^{3}$ Max Planck Institute for Dynamics and Self-Organization, Am Fassberg 17, \\ 37077 Göttingen, Germany
}

(Received 8 April 2018; revised 29 May 2018; accepted 20 June 2018;

first published online 20 July 2018)

We experimentally study the influence of wall roughness on bubble drag reduction in turbulent Taylor-Couette flow, i.e. the flow between two concentric, independently rotating cylinders. We measure the drag in the system for the cases with and without air, and add roughness by installing transverse ribs on either one or both of the cylinders. For the smooth-wall case (no ribs) and the case of ribs on the inner cylinder only, we observe strong drag reduction up to $\mathrm{DR}=33 \%$ and $\mathrm{DR}=23 \%$, respectively, for a void fraction of $\alpha=6 \%$. However, with ribs mounted on both cylinders or on the outer cylinder only, the drag reduction is weak, less than $\mathrm{DR}=11 \%$, and thus quite close to the trivial effect of reduced effective density. Flow visualizations show that stable turbulent Taylor vortices - large-scale vortical structures - are induced in these two cases, i.e. the cases with ribs on the outer cylinder. These strong secondary flows move the bubbles away from the boundary layer, making the bubbles less effective than what had previously been observed for the smooth-wall case. Measurements with counter-rotating smooth cylinders, a regime in which pronounced Taylor rolls are also induced, confirm that it is really the Taylor vortices that weaken the bubble drag reduction mechanism. Our findings show that, although bubble drag reduction can indeed be effective for smooth walls, its effect can be spoiled by e.g. biofouling and omnipresent wall roughness, as the roughness can induce strong secondary flows.

Key words: drag reduction, multiphase flow, Taylor-Couette flow

\section{Introduction}

In the maritime industry, air lubrication is seen as one of the most promising techniques to reduce the overall fuel consumption (Kodama et al. 2000; Foeth 2008;

†Email addresses for correspondence: chaosun@tsinghua.edu.cn, d.lohse@utwente.nl 
Mäkiharju, Perlin \& Ceccio 2012). Air lubrication has been studied for several decades, and it is found that a few per cent of air can significantly decrease the overall friction, e.g. with $4 \%$ bubbles, drag reductions up to $40 \%$ were shown (van Gils et al. 2013; Verschoof et al. 2016). Notwithstanding its clear industrial potential, it remains difficult to translate highly controlled laboratory results to drag reduction in large-scale vessels. So far, the vast majority of studies on bubble drag reduction (DR) have been performed in test facilities with purified water and smooth walls: see e.g. the review articles by Ceccio (2010) and Murai (2014). However, many surfaces in industry are rough to some extent, and also initially smooth surfaces can become rough by means of corrosion, cavitation, mineral scaling and (bio)fouling (Schultz 2007; Schultz et al. 2011). Furthermore, the dynamics of bubbles is strongly affected by any dissolved ions in oceanic water and surfactants (Takagi, Ogasawara \& Matsumoto 2008; Takagi \& Matsumoto 2011). As the conditions in controlled experiments and real applications are so different, one can expect that bubble DR experiments will lead to very different results in practice. Only a limited number of studies focused on 'non-ideal' DR, either through wall roughness (Deutsch et al. 2004; Van den Berg et al. 2007; Elbing et al. 2008, 2013), surfactants or seawater (Takahashi et al. 2001; Winkel et al. 2004; Shen, Perlin \& Ceccio 2006; Elbing et al. 2008; Verschoof et al. 2016), and their results are somewhat inconsistent. Some studies found that wall roughness completely eliminates any drag reduction (Van den Berg et al. 2007). Another study with rough-wall flat plate measurements showed that bubble DR or air layer DR can still be effective, at the cost, however, of an increased required air injection flux as the roughness forces the air away from the wall (Elbing et al. 2013). A third study showed that roughness can even enhance drag reduction (Deutsch et al. 2004). Given this variety of conclusions, there is a clear need to better understand the influence of wall roughness on bubble drag reduction.

In this work, we study the effect of wall roughness on bubble drag reduction. To do so, we employ the Taylor-Couette (TC) system, i.e. the flow between two concentric, independently rotating cylinders (Fardin, Perge \& Taberlet 2014; Grossmann, Lohse \& Sun 2016). TC flow is one of the canonical flow systems in which fluid mechanics concepts and theories are tested. Among the advantages of using a TC set-up are the ease with which the global void fraction $\alpha$ is controlled, the absence of any streamwise spatial transients and, as it is a closed system, an exact balance that connects the global torque measurements with the local energy dissipation rate. The driving and response of the system are characterized by the Taylor number $\mathrm{Ta}$ and the Nusselt number $N u_{\omega}$, respectively (Grossmann et al. 2016). The Nusselt number is defined as the ratio of the convective momentum transport to the diffusive flux, and using it underlines the close analogy between Taylor-Couette flow and Rayleigh-Bénard convection (Eckhardt, Grossmann \& Lohse 2007). In the currently studied parameter regime, an effective scaling of $N u_{\omega} \propto T a^{0.4}$ is observed (van Gils et al. 2011b). The TC set-up has been used frequently to study (bubble) drag reduction in turbulent flows (van den Berg et al. 2005; van Gils et al. 2013; Rosenberg et al. 2016; Saranadhi et al. 2016; Verschoof et al. 2016), even numerically (Sugiyama, Calzavarini \& Lohse 2008; Spandan, Verzicco \& Lohse 2017). In these studies, it was shown that a small air fraction can considerably reduce the drag. For example, with a void fraction of $\alpha=4 \%$, a drag reduction of $40 \%$ was observed (van Gils et al. 2013; Verschoof et al. 2016), which is significantly larger than the trivial effects of affected effective density and viscosity. These studies highlighted the importance of bubble deformability for large drag reduction, and thus a sufficiently large Weber number $W e=\rho D_{b} u^{2} / \sigma$, in which $\rho$ is the fluid density, $D_{b}$ 
the bubble diameter, $u$ a characteristic velocity and $\sigma$ the interfacial surface tension. It was shown that large Weber number bubbles, i.e. large and deformable bubbles, are crucial to efficiently reduce the drag. The bubbles experience multiple forces. Most important are (i) centripetal forces, pushing air towards the inner cylinder, (ii) buoyancy forces, pushing air towards the top and (iii) turbulent fluctuations, mixing the bubbles throughout the flow. Large bubbles are hardly affected by the turbulent fluctuations, and are therefore strongly pushed towards the inner cylinder. The role of the buoyancy forces decreases with increasing rotation rates. Wall roughness, on the other hand, obviously increases the friction. Its effects have been studied extensively for single-phase turbulence flows, mostly in pipe or channel flow configurations given their industrial relevance: see e.g. Marusic et al. (2010), Flack \& Schultz (2014) and references therein. For TC flow, adding sufficiently large, rough ribs results in a $N u_{\omega} \propto T a^{1 / 2}$ scaling, rather than the aforementioned $N u_{\omega} \propto T a^{0.4}$ smooth-wall scaling (Cadot et al. 1997; van den Berg et al. 2003; Zhu et al. 2018). In this regime, the main contribution to the torque originates from the pressure force acting on the ribs rather than the skin friction on the cylinder (Zhu et al. 2018). The $N u_{\omega} \propto T a^{1 / 2}$ scaling, mathematically equivalent to a constant friction coefficient in the fully rough regime, is the mathematical upper bound to the transport of momentum. In this regime, the roughness decreases the near-wall velocity gradient, whereas the streamwise velocity fluctuations are increased. The bubble dynamics is largely governed by the motion of the surrounding fluid, but to which extent any drag reduction is affected by the changed fluid motion is unknown.

A number of studies focused on wall modifications to stimulate air to attach to the inner cylinder wall of Taylor-Couette flow, either by a hydrophobic coating (Srinivasan et al. 2015) or by using cavitors to try to create an air layer (Verschoof et al. 2018). Van den Berg et al. (2007) studied the effects of roughness on bubble drag reduction, and found that ribs attached to both cylinders prevent bubbles from reducing the overall friction. Therefore, it was suggested that bubbly drag reduction is a boundary layer effect. However, the exact reason why the drag reduction was lost remained elusive. Therefore, here we aim to repeat and extend those experiments in a more accurate and controlled set-up and to visualize the flow, to better understand the physics of the aforementioned conclusions.

\section{Experimental method}

The experiments are performed in the Twente Turbulent Taylor-Couette facility $\left(\mathrm{T}^{3} \mathrm{C}\right.$ ) (van Gils et al. 2011a), in which the flow is fully turbulent. TC flow is driven by the angular velocity of the inner and outer cylinder, denoted by $\omega_{i}$ and $\omega_{o}$, respectively. The set-up has a height of $L=927 \mathrm{~mm}$, an inner radius $r_{i}=200 \mathrm{~mm}$ and an outer radius $r_{o}=279.4 \mathrm{~mm}$, giving a gap width $d=r_{o}-r_{i}=79.4 \mathrm{~mm}$. The geometry can therefore be described by two geometric parameters: the radius ratio $\eta=r_{i} / r_{o}=0.716$ and the aspect ratio $\Gamma=L / d=11.7$; see also figure $1(a)$. The inner cylinder and outer cylinder rotate up to $f_{i}=\omega_{i} /(2 \pi)=10 \mathrm{~Hz}$ and $f_{o}=-4 \mathrm{~Hz}$, respectively. These result in two Reynolds numbers, $R e_{i, o}=\omega_{i, o} r_{i, o}\left(r_{o}-r_{i}\right) / v$, respectively, in which $v$ is the viscosity of the working fluid. The rotation ratio $a$ is defined as $a=-\omega_{o} / \omega_{i}$. We here express the driving using the Taylor number $T a=\left[(1+\eta)^{4} /\left(64 \eta^{2}\right)\right] d^{2}\left(r_{i}+r_{o}\right)^{2}\left(\omega_{i}-\omega_{o}\right)^{2} v^{-2} \propto\left(R e_{i}-\eta R e_{o}\right)^{2}$, which thus incorporates the rotation of both cylinders in one dimensionless number. In the current study, we measure at Taylor numbers of $O\left(10^{12}\right)$, or, equivalently, Reynolds numbers up to $O\left(10^{6}\right)$. The primary response parameter is the torque $\tau$ necessary to maintain 
(a)

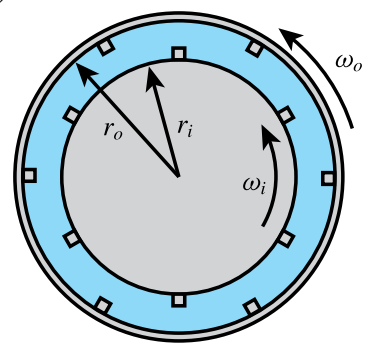

(b)

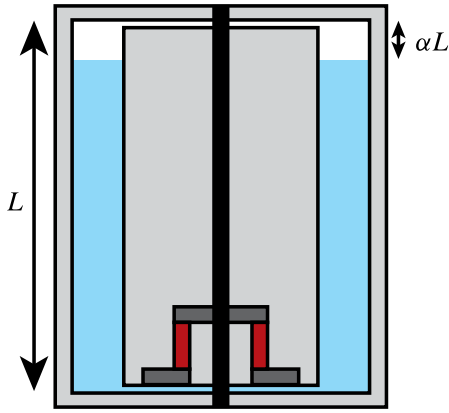

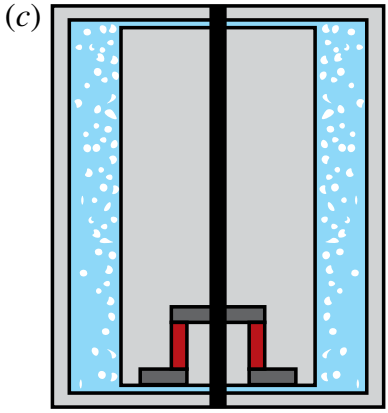

FIgURE 1. (Colour online) Experimental set-up. (a) Top view schematic of the $\mathrm{T}^{3} \mathrm{C}$ facility. We attached six vertical transverse ribs (not to scale) equally distributed around the perimeter of the inner cylinder, the outer cylinder or both cylinders. We also measure a smooth-wall case without any ribs. (b) Vertical cross-section of the set-up at rest, showing the position of the torque sensor. To control the void fraction, we fill the cylinder only partially with water, so that the void fraction $\alpha$ is controlled by measuring the relative height of the water level. (c) Vertical cross-section of the set-up during a measurement. The free surface disappears, and all air is entrained by the turbulent flow (bubbles not to scale).

the inner cylinder at a constant angular velocity. The torque is measured with a co-axial torque transducer (Honeywell Hollow Reaction Torque Sensor 2404-1K, maximum capacity of $115 \mathrm{Nm}$ ), which is located inside the inner cylinder to avoid measurement errors due to seal and bearing friction: see figure $1(b)$. The torque is made dimensionless with the torque for laminar non-vertical flow, resulting in the Nusselt number $N u_{\omega}=\tau / \tau_{\text {lam }}$, with $\tau_{\text {lam }}=4 \pi L \rho v r_{i}^{2} r_{o}^{2}\left(\omega_{i}-\omega_{o}\right) /\left(r_{o}^{2}-r_{i}^{2}\right)$. The flow is cooled through both endplates to counteract viscous heating, keeping the water temperature constant within $T=21 \pm 0.5{ }^{\circ} \mathrm{C}$. Although the effective viscosity and density are altered by the presence of bubbles, we chose to consequently use the pure water material properties, as we are interested in the net changes in drag.

The cylinders are made rough by attaching six transverse ribs over the entire height of the cylinders, as shown in figure $1(a)$. The rib dimensions are $6 \mathrm{~mm}$ by $6 \mathrm{~mm}$, corresponding to $7.5 \%$ of the gap width, and to $O\left(10^{3}\right)$ in wall units, depending on the Taylor number and roughness case. We study the torque and resulting drag reduction for four cases: both cylinders smooth (SS), both cylinders rough (RR), and roughness either only on the inner cylinder (RS) or only on the outer cylinder (SR). We here chose to apply rib roughness, which, for the RR case, causes the flow to be in the 'fully rough' state, or the 'asymptotic ultimate turbulence' regime (Zhu et al. 2018) in the studied parameter regime. In this regime, the behaviour in the boundary layers becomes independent of the viscosity. Consequently, a $N u_{\omega} \propto T a^{1 / 2}$ is observed rather than the effective $N u_{\omega} \propto T a^{0.4}$ scaling found for the smooth-wall case in the currently studied parameter regime (Kraichnan 1962; van Gils et al. 2011b; Zhu et al. 2018). For the cases of ribs on a single cylinder, the exponent $\gamma$ of the $N u_{\omega} \propto T a^{\gamma}$-scaling is between these two bounds.

The gap is either partially or completely filled with water, so that the void fraction is precisely set at $0 \% \leqslant \alpha \leqslant 6 \%$ : see figure $1(b)$. We determine the void fraction with both cylinders at rest. During a flow measurement, the air is distributed over the height of the cylinder because of turbulent mixing: see figure 1(c). The uncertainty in the void fraction $\alpha$ is around $\pm 0.2 \%$. We note that a perfect homogeneous axial 

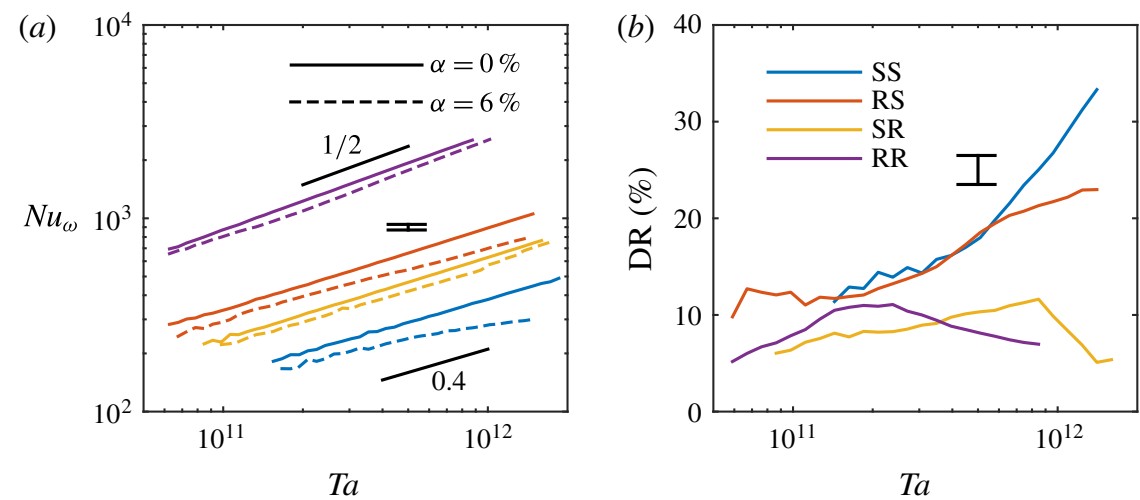

FIgURE 2. (Colour online) (a) Dimensionless angular velocity flux $N u_{\omega}$ as a function of $T a$ for $\alpha=0 \%$ and $\alpha=6 \%$. To increase readability, we do not show $N u_{\omega}$ for $\alpha=$ $\{2 \%, 4 \%\}$, which are used to calculate the DR shown in figure 3 . The two short black lines indicate the $N u_{\omega} \propto T a^{\gamma}$ scaling relations for the pure liquid cases. The exponents are $\gamma=0.4$ and $\gamma=1 / 2$ for the SS and RR cases, respectively. (b) Resulting drag reduction as a function of Ta. The outer cylinder is stationary. The DR is calculated with (3.1). Typical error bars are shown.

distribution is not feasable, even with continuous bubble injection through the bottom end cap (as was shown in van Gils et al. (2013)), but it becomes more homogeneous with increasing Taylor number.

\section{Results}

We measure the torque and present our findings in figures 2 and 3 . The drag reduction is calculated as

$$
\mathrm{DR}=1-\frac{N u_{\omega}(\alpha)}{N u_{\omega}(\alpha=0)},
$$

in which we compare the $N u_{\omega}$ values for the same roughness case. In figure 2, we show the Nusselt number and resulting drag reduction for all roughness cases. As was shown before, $N u_{\omega}$ depends tremendously on the applied roughness (Zhu et al. 2018). In the current study, however, we are more interested in the relative bubbly drag reduction as compared to the smooth-wall case, rather than the absolute friction increase by roughness. From figure 2 two different regimes can be distinguished: we observe strong drag reduction for the SS and RS cases, up to DR $=33 \%$ with a void fraction of $\alpha=6 \%$, whereas for the SR and RR cases, the DR is only weak, with the same void fraction never exceeding $\mathrm{DR}=12 \%$.

To study the DR per roughness case further, we show the drag reduction for void fractions of $2 \%, 4 \%$ and $6 \%$ in figure 3. The DR increased monotonically with increasing void fraction for all cases. In the weak drag reduction cases (SR and RR), the DR is quite close to the trivial effect of reduced global density, which equals $\rho_{\text {eff }}=\rho(1-\alpha)+\alpha \rho_{\text {air }} \approx \rho(1-\alpha)$, in which $\rho_{\text {air }}$ is the air density. For the RS and SS cases, however, the drag reduction is significantly larger than the reduced density effect. Interestingly, given the strong DR in the RS case, it is clear that wall roughness does not necessarily prevent strong bubble drag reduction. In fact, ribs in the RS case have a similar effect on the flow as cavitators used in air cavity DR 

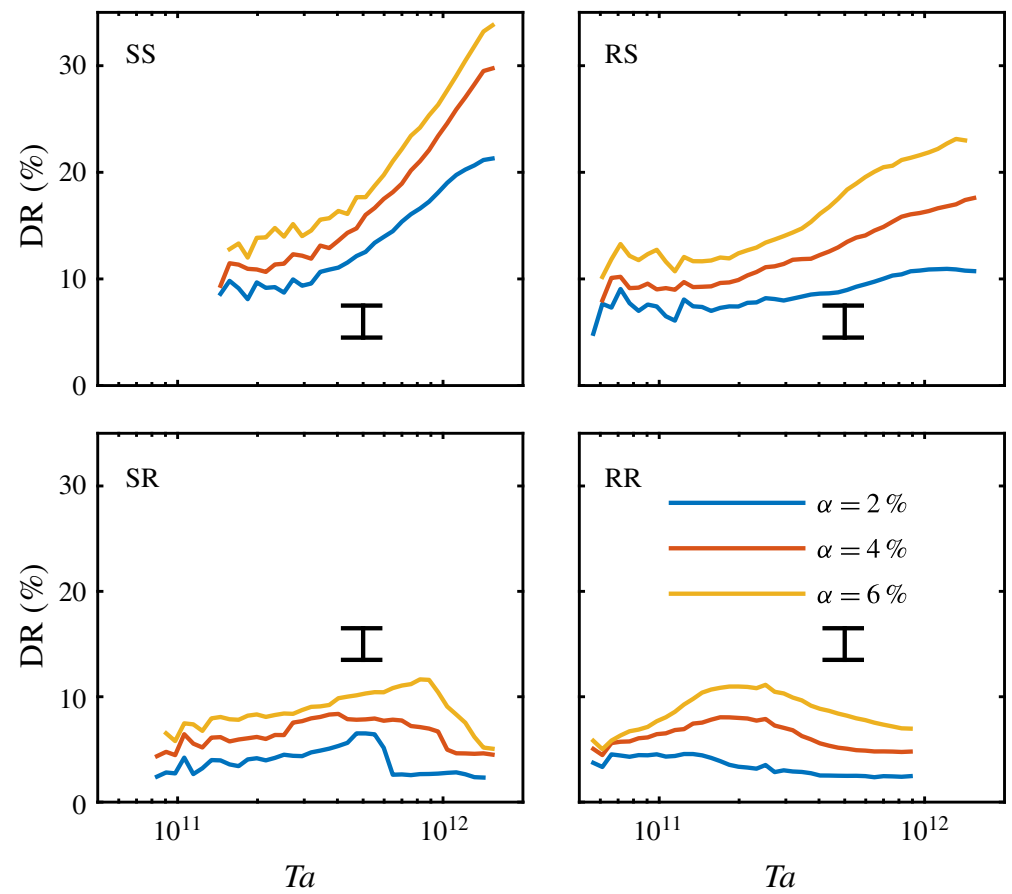

FIGURE 3. (Colour online) Drag reduction percentages as a function of $T a$ for all roughness cases. In the RR case, the highest achievable Taylor number is slightly smaller due to experimental limitations. The outer cylinder is stationary. The DR is calculated with (3.1). Typical error bars are shown.

(Zverkhovskyi 2014; Verschoof et al. 2018). The ribs create a low pressure region in the wake, attracting air to form an air layer. In the RS case, both bubble DR and air cavity DR contribute to the effective DR. For both the RS and SS cases, the drag reduction increases with Taylor number (van Gils et al. 2013), contrasting the SR and $\mathrm{RR}$ cases, in which the drag reduction does not have a clear monotonic Taylor number dependence.

To better understand the flow dynamics, we visualize the flow for a void fraction of $\alpha=6 \%$. As shown in figure 4 , for all four cases the flow structures are significantly different. In the SS and RS cases, clear streaks and patterns are visualized by the bubbles, but stable turbulent Taylor vortices are not observed. For both cases with ribs on the outer cylinder, i.e. the SR and RR cases, we do however observe stable turbulent Taylor vortices.

The existence and the dynamics of Taylor vortices have been studied extensively for the single-phase smooth-wall case (Lathrop, Fineberg \& Swinney 1992a,b; Lewis \& Swinney 1999; Huisman et al. 2014; Grossmann et al. 2016; van der Veen et al. 2016). In the explored Taylor number regime, measurements showed that for sufficiently strong turbulence $\left(R e_{i}>10^{5}\right)$, stable Taylor rolls do not exist in the pure inner cylinder rotation regime, and are only present in the counter-rotating regime (Ostilla-Mónico et al. 2014; Grossmann et al. 2016). Roughness elements promote the ejection of turbulent plumes, leading to localized radial flows towards the outer cylinder (Zhu et al. 2016; Toppaladoddi, Succi \& Wettlaufer 2017). As the TC system is closed, consequently a radial flow towards the inner cylinder must 

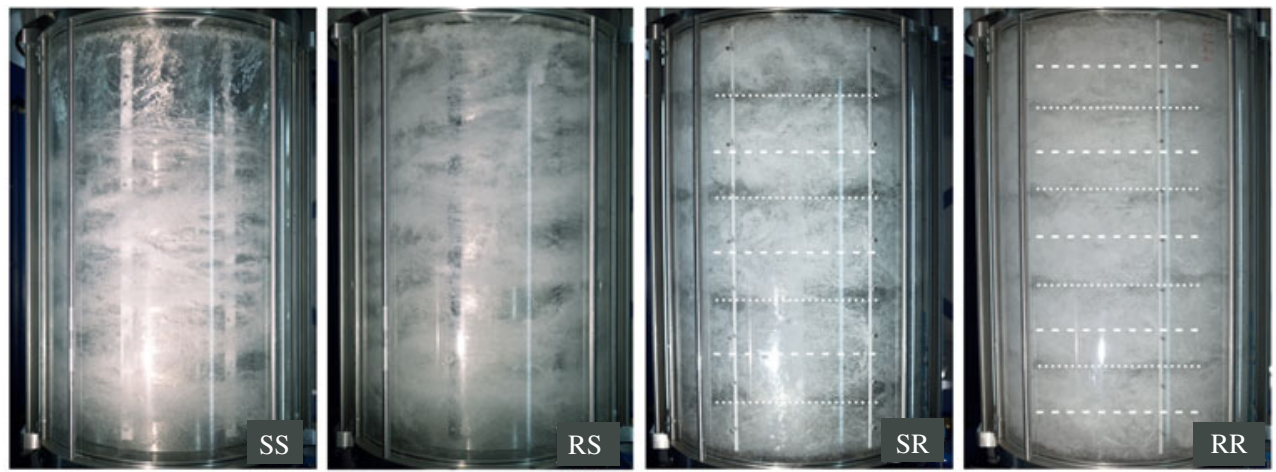

FIGURE 4. (Colour online) Instantaneous photographs of the flow for all four roughness cases: SS, RS, SR and RR. Clear differences in the flow patterns are visible. In the SS and RS cases, we see turbulent streaks, but no stable structures. Clear stable Taylor rolls are visible for the SR and RR cases. We indicate the position of the rolls with the dashed line and the roll pairs with the dotted line. The Taylor number is $T a=1.5 \times 10^{12}$, except for the RR case $\left(T a=8.4 \times 10^{11}\right)$, while the outer cylinder is kept stationary. The void fraction in all cases is $\alpha=6 \%$. Note that in all cases the bubbles are not homogeneously distributed over the height; this is most visible in the SS case.

be present. These flows can organize themselves as stable Taylor rolls. Thus, as the roughness promotes the ejection of turbulent plumes, the existence of Taylor vortices is stimulated.

The roll dynamics observed with wall roughness is different from what has been observed hitherto in the same set-up. Earlier studies found six or eight rolls for the smooth-wall case with counter-rotating cylinders (Huisman et al. 2014; van der Veen et al. 2016). Here, for pure inner cylinder rotation, we see ten rolls for the RR case, whereas for the SR case we observe eight rolls. The number of rolls is related to the aspect ratio $\Gamma$, which depends on the gap width. The roughness elements decrease the 'effective gap width', and thus increase the apparent aspect ratio $\Gamma_{\text {eff }}$, which is calculated by subtracting the rib height from the gap width. This results in aspect ratios of $\Gamma_{S R, R S}=L /(d-h)=12.7$ and $\Gamma_{R R}=L /(d-2 h)=13.8$, whereas $\Gamma=L / d=$ 11.7. Consequently, the system allows for an increased number of rolls in the roughwall cases. A roll aspect ratio can be defined as $A R=\Gamma / N_{r}$, in which $N_{r}$ is the number of rolls (Chouippe et al. 2014; Huisman et al. 2014; van der Veen et al. 2016). With the current radius ratio, the smooth wall roll aspect ratios are found to be $1.14 \leqslant$ $A R_{S S} \leqslant 1.96$, with a value of $A R_{S S}=1.46$ being most common in the currently used set-up. In the SR and RR cases, we observe eight and ten rolls, respectively, resulting in roll aspect ratios of $A R_{S R}=1.59$ and $A R_{R R}=1.38$, respectively.

We argue that the existence of the Taylor vortices is the underlying mechanism through which the effectiveness of bubble drag reduction is reduced in the SR and RR cases. The Taylor vortices have two significant effects on the air: (i) the vortices enhance turbulent mixing of the flow, and (ii) the vortices attract air to the vortex core, as the local pressure in the core of the Taylor vortices is low. To effectively decrease the drag, it is crucial that large bubbles are present in or close to the boundary layer (van Gils et al. 2013; Verschoof et al. 2016; Spandan et al. 2017). The flow visualizations show that the bubbles are dragged away from the inner cylinder wall, and are radially more mixed. Therefore, as the bubbles do not accumulate close to 


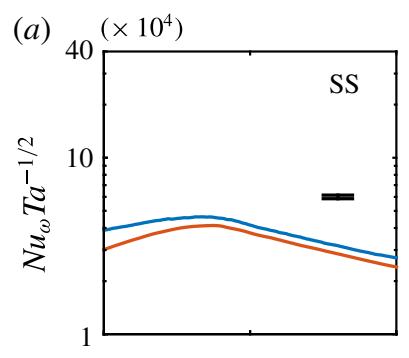

(b)

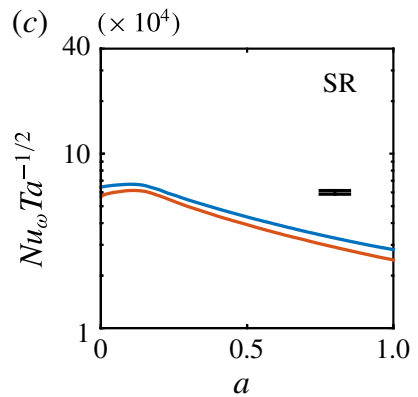

(d)
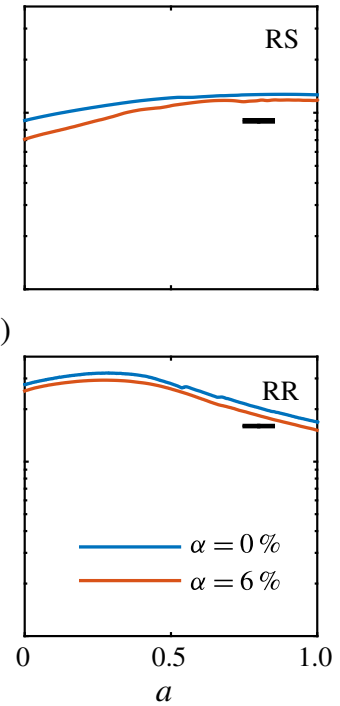

(e)

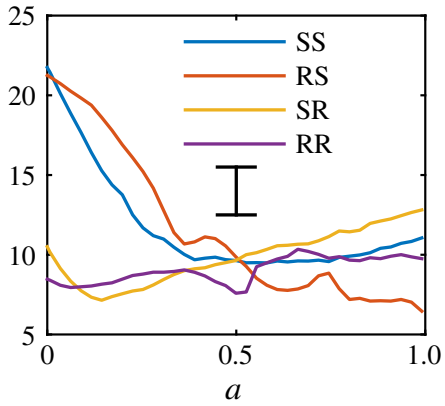

FIGURE 5. (Colour online) $(a-d)$ Dimensionless torque as a function of rotation ratio $a$ for the roughness cases SS, SR, RS and RR with and without air. The Taylor number is kept constant at $T a=7.3 \times 10^{11}$. We compensate $N u_{\omega}$ by $T a^{0.5}$ to remove viscosity changes due to temperature fluctuations, similar to Huisman et al. (2014). (e) Resulting drag reduction as a function of rotation ratio $a$, calculated here as $\mathrm{DR}=1-$ $\left[N u_{\omega} T^{-0.4}(\alpha=6 \%)\right] /\left[N u_{\omega} T^{-0.4}(\alpha=0 \%)\right]$. The rotation ratio was increased from $a=0$ to $a=1$ in a single, continuous quasi-static measurement. For figure $(b)$, we binned the results using 40 linearly distributed bins. Typical error bars are shown.

the inner cylinder, the drag reduction almost vanishes, and becomes close to the trivial effect of the reduced global effective density, as was shown in our torque measurements.

Up to here, we showed that in the SR and RR cases, the ribs induce turbulent Taylor vortices, and we argued that Taylor rolls eliminate DR. One could presume that the rolls, instead of being the underlying physical explanation, merely coincide with the weak DR. To further prove the effect of turbulent Taylor vortices on bubble DR, we study the DR behaviour in the counter-rotation regime. For the SS case, pronounced stable turbulent Taylor vortices exist in the counter-rotating regime within approximately $0.1 \leqslant a \leqslant 0.5$ (van Gils et al. 2012; Huisman et al. 2014; van der Veen et al. 2016). By measuring the DR as a function of rotation ratio $a=-\omega_{o} / \omega_{i}$ while keeping the Taylor number constant, we can directly show the influence of Taylor rolls on the effectiveness of air lubrication. For all roughness cases, we show the torque in figure $5(a-d)$ and the resulting DR in figure 5(e). As already shown before, we observe strong DR for the SS and SR cases at $a=0$. Then, for increasing $a$, we see that the DR decreases. The observation is very similar to the previously discussed weak DR in the SR and RR cases, namely in the counter-rotating regime the bubbles are trapped in the Taylor rolls, dragged away from the boundary layer and unable to effectively decrease the drag. For the SR case, the strength of the turbulent Taylor vortices decreases with increasing outer cylinder. Consequently, an increase in DR is observed. For the RR case, the DR remains weak for all cases, as the turbulent Taylor vortices exist for the entire scanned parameter space. 


\section{Conclusions}

To conclude, we studied the influence of wall roughness on bubble drag reduction in a highly turbulent flow. We showed that in the SR and RR cases wall roughness promotes stable turbulent Taylor rolls, which induce strong secondary flows, suppressing the drag reduction. Bubbles are captured in low vorticity regions, and therefore dragged away from the inner cylinder boundary layer. As a result, the drag reduction is mostly lost, and the effective drag reduction is close to the trivial effect of reduced global density. These findings help us to understand earlier studies on air lubrication and wall roughness, which had conflicting results as to whether roughness influences bubble drag reduction. We here distinguish two different regimes: (i) a regime with strong drag reduction if the roughness does not introduce strong secondary flows, and (ii) a regime with weak drag reduction if strong secondary flows are induced by the roughness.

Future work will include studies on wall roughness combined with bubbles in other types of set-ups, e.g. flat plates, or pipe flow. In these set-ups, roughness increases the velocity fluctuations but does not necessarily induce stable large-scale secondary flows, and thus the bubble DR behaviour might be significantly different from that in the current study. Moreover, as we limited ourselves to the influence of rib roughness, more work is needed to understand the influence of more realistic types of roughness.

\section{Acknowledgements}

We thank Tom van Terwisga (MARIN, TU Delft) for the continuous and stimulating collaboration over the years on drag reduction in the marine context. We also thank Dennis van Gils, Gert-Wim Bruggert and Martin Bos for their technical support. The work was financially supported by NWO-TTW (project 13265). We acknowledge support from EuHIT and MCEC. C.S. and D.B. acknowledge financial support from VIDI grant no. 13477, and the Natural Science Foundation of China under grant no. 11672156. P.A.B acknowledges NWO-TTW (project 14504).

\section{REFERENCES}

Van den Berg, T. H., Doering, Ch. R., Lohse, D. \& Lathrop, D. P. 2003 Smooth and rough boundaries in turbulent Taylor-Couette flow. Phys. Rev. E 68, 036307.

Van den Berg, T. H., van Gils, D. P. M., Lathrop, D. P. \& Lohse, D. 2007 Bubbly turbulent drag reduction is a boundary layer effect. Phys. Rev. Lett. 98, 084501.

VAn Den Berg, T. H., Luther, S., Lathrop, D. P. \& Lohse, D. 2005 Drag reduction in bubbly Taylor-Couette turbulence. Phys. Rev. Lett. 94, 044501.

Cadot, O., Couder, Y., Daerr, A., Doundy, S. \& Tsinober, A. 1997 Energy injection in closed turbulent flows: stirring through boundary layers versus inertial stirring. Phys. Rev. E 56, 427-433.

Ceccio, S. L. 2010 Friction drag reduction of external flows with bubble and gas injection. Annu. Rev. Fluid Mech. 42, 183-203.

Chouippe, A., Climent, E., Legendre, D. \& Gabillet, C. 2014 Numerical simulation of bubble dispersion in turbulent Taylor-Couette flow. Phys. Fluids 26 (4), 043304.

Deutsch, S., Moeny, M., Fontaine, A. A. \& Petrie, H. 2004 Microbubble drag reduction in rough walled turbulent boundary layers with comparison against polymer drag reduction. Exp. Fluids 37, 731-744.

Eckhardt, B., Grossmann, S. \& Lohse, D. 2007 Torque scaling in turbulent Taylor-Couette flow between independently rotating cylinders. J. Fluid Mech. 581, 221-250. 
Elbing, B. R., Mäkiharju, S., Wiggins, A., Perlin, M., Dowling, D. R. \& Ceccio, S. L. 2013 On the scaling of air layer drag reduction. J. Fluid Mech. 717, 484-513.

Elbing, B. R., Winkel, E. S., Lay, K. A., Ceccio, S. L., Dowling, D. R. \& Perlin, M. 2008 Bubble-induced skin-friction drag reduction and the abrupt transition to air-layer drag reduction. J. Fluid Mech. 612, 201-236.

Fardin, M. A., Perge, C. \& Taberlet, N. 2014 'The hydrogen atom of fluid dynamics': introduction to the Taylor-Couette flow for soft matter scientists. Soft Matt. 10, 3523.

Flack, K. A. \& Schultz, M. P. 2014 Roughness effects on wall-bounded turbulent flows. Phys. Fluids 26 (10), 101305.

Foeth, E. J. 2008 Decreasing frictional resistance by air lubrication. In 20th Int. HISWA Symp. on Yacht Design and Yacht Construction. HISWA.

van Gils, D. P. M., Bruggert, G. W., Lathrop, D. P., Sun, C. \& Lohse, D. $2011 a$ The Twente turbulent Taylor-Couette $\left(\mathrm{T}^{3} \mathrm{C}\right)$ facility: strongly turbulent (multi-phase) flow between independently rotating cylinders. Rev. Sci. Instrum. 82, 025105.

van Gils, D. P. M., Huisman, S. G., Bruggert, G. W., Sun, C. \& Lohse, D. $2011 b$ Torque scaling in turbulent Taylor-Couette flow with co- and counter-rotating cylinders. Phys. Rev. Lett. 106, 024502.

van Gils, D. P. M., Huisman, S. G., Grossmann, S., Sun, C. \& Lohse, D. 2012 Optimal Taylor-Couette turbulence. J. Fluid Mech. 706, 118-149.

van Gils, D. P. M., Narezo Guzman, D., Sun, C. \& Lohse, D. 2013 The importance of bubble deformability for strong drag reduction in bubbly turbulent Taylor-Couette flow. J. Fluid Mech. 722, 317-347.

Grossmann, S., Lohse, D. \& Sun, C. 2016 High Reynolds number Taylor-Couette turbulence. Annu. Rev. Fluid Mech. 48, 53.

Huisman, S. G., van der Veen, R. C. A., Sun, C. \& Lohse, D. 2014 Multiple states in highly turbulent Taylor-Couette flow. Nat. Commun. 5, 3820.

Kodama, Y., Kakugawa, A., Takahashi, T. \& Kawashima, H. 2000 Experimental studies on microbubbles and their applicability to ships for skin friction reduction. Intl J. Heat Fluid Flow 21, 582-588.

Kraichnan, R. H. 1962 Turbulent thermal convection at arbritrary Prandtl number. Phys. Fluids 5, 1374.

Lathrop, D. P., Fineberg, J. \& Swinney, H. S. $1992 a$ Transition to shear-driven turbulence in Couette-Taylor flow. Phys. Rev. A 46, 6390-6405.

Lathrop, D. P., Fineberg, J. \& Swinney, H. S. $1992 b$ Turbulent flow between concentric rotating cylinders at large Reynolds numbers. Phys. Rev. Lett. 68, 1515-1518.

LEwis, G. S. \& Swinney, H. L. 1999 Velocity structure functions, scaling, and transitions in high-Reynolds-number Couette-Taylor flow. Phys. Rev. E 59, 5457-5467.

MÄKiharju, S., Perlin, M. \& Ceccio, S. L. 2012 On the energy economics of air lubrication drag reduction. Intl. J. Nav. Archit. Ocean Engng. 4, 412-422.

Marusic, I., McKeon, B. J., Monkewitz, P. A., Nagib, H. M., Smits, A. J. \& Sreenivasan, K. R. 2010 Wall-bounded turbulent flows at high Reynolds numbers: recent advances and key issues. Phys. Fluids 22 (6), 065103.

Murai, Y. 2014 Frictional drag reduction by bubble injection. Exp. Fluids 55 (7), 1773.

Ostilla-Mónico, R., van der Poel, E. P., Verzicco, R., Grossmann, S. \& Lohse, D. 2014 Exploring the phase diagram of fully turbulent Taylor-Couette flow. J. Fluid Mech. 761, 1-26.

Rosenberg, B. J., van Buren, T., Matthew, K. F. \& Smits, A. J. 2016 Turbulent drag reduction over air- and liquid-impregnated surfaces. Phys. Fluids 28, 015103.

Saranadhi, D., Chen, D., Kleingartner, J. A., Srinivasan, S., Cohen, R. B.\& McKinley, G. H. 2016 Sustained drag reduction in a turbulent flow using a low temperature Leidenfrost surface. Sci. Adv. 2 (10), E1600686.

SCHUlTZ, M. P. 2007 Effects of coating roughness and biofouling on ship resistance and powering. Biofouling 23 (5), 331-341.

Schultz, M. P., Bendick, J. A., Holm, E. R. \& Hertel, W. M. 2011 Economic impact of biofouling on a naval surface ship. Biofouling 27 (1), 87-98. 
Shen, X., Perlin, M. \& Ceccio, S. L. 2006 Influence of bubble size on micro-bubble drag reduction. Exp. Fluids 41, 415-424.

Spandan, V., Verzicco, R. \& Lohse, D. 2017 Deformable ellipsoidal bubbles in Taylor-Couette flow with enhanced Euler-Lagrangian tracking. Phys. Rev. Fluids 2, 104304.

Srinivasan, S., Kleingartner, J. A., Gilbert, J. B., Cohen, R. B., Milne, A. J. B. \& MCKinley, G. H. 2015 Sustainable drag reduction in turbulent Taylor-Couette flows by depositing sprayable superhydrophobic surfaces. Phys. Rev. Lett. 114, 014501.

Sugiyama, K., Calzavarini, E. \& Lohse, D. 2008 Microbubble drag reduction in Taylor-Couette flow in the wavy vortex regime. J. Fluid Mech. 608, 21-41.

TAKagi, S. \& Matsumoto, Y. 2011 Surfactant effects on bubble motion and bubbly flows. Annu. Rev. Fluid Mech. 43 (1), 615-636.

Takagi, S., Ogasawara, T. \& Matsumoto, Y. 2008 The effects of surfactant on the multiscale structure of bubbly flows. Phil. Trans. A. Math. Phys. Engng Sci. 366 (1873), 2117-2129.

Takahashi, T., Kakugawa, A., Nagaya, S., Yanagihara, T. \& Kodama, Y. 2001 Mechanisms and scale effects of skin friction reduction by microbubbles. In Proceedings of 2nd Symposium on Smart Control of Turbulence, University of Tokyo.

Toppaladoddi, S., Succi, S. \& Wettlaufer, J. S. 2017 Roughness as a route to the ultimate regime of thermal convection. Phys. Rev. Lett. 118, 074503.

Van der Veen, R. C. A., Huisman, S. G., Dung, O.-Y., Tang, H.-L., Sun, C. \& Lohse, D. 2016 Exploring the phase space of multiple states in highly turbulent Taylor-Couette flow. Phys. Rev. Fluids 1, 024401.

Verschoof, R. A., Bakhuis, D., Bullee, P. A., Huisman, S. G., Sun, C. \& Lohse, D. 2018 Air cavities at the inner cylinder of turbulent Taylor-Couette flow. Intl J. Multiphase Flow 105, 264-273.

Verschoof, R. A., van der Veen, R. C. A., Sun, C. \& Lohse, D. 2016 Bubble drag reduction requires large bubbles. Phys. Rev. Lett. 117, 104502.

Winkel, E. S., Ceccio, S. L., Dowling, D. R. \& Perlin, M. 2004 Bubble-size distributions produced by wall injection of air into flowing freshwater, saltwater and surfactant solutions. Exp. Fluids 37, 802-810.

Zhu, X., Ostilla-Monico, R., Verzicco, R. \& Lohse, D. 2016 Direct numerical simulation of Taylor-Couette flow with grooved walls: torque scaling and flow structure. J. Fluid Mech. 794, 746-774.

Zhu, X., Verschoof, R. A., Bakhuis, D., Huisman, S. G., Verzicco, R., Sun, C. \& Lohse, D. 2018 Wall roughness induces asymptotic ultimate turbulence. Nat. Phys. 14, 417-423.

Zverkhovskyi, O. 2014 Ship drag reduction by air cavities. PhD thesis, Delft University of Technology, Delft, NL. 